\title{
La Storia morantiana sullo schermo
}

\author{
Hanna SERKOWSKA ${ }^{1}$ \\ Università di Varsavia \\ hanna.serkowska@uw.edu.pl
}

\begin{abstract}
RIASSUNTO
Questa lettura dell'adattamento della Storia, fatto da Luigi Comencini per il piccolo schermo, è avulsa alla tesi su una sostanziale risemantizzazione del testo letterario. Si dimostra che questo regista, autore di cinema morale e pedagogico, ma avverso all'uso del cinema ai fini politici (e attento a non cadere nel miserabilismo neorealista), accoglie fedelmente gli intenti morantiani. Fa un film sull'innocenza minacciata dalla morte e dalla violenza in tempo della guerra (così come essa è soffocata dall'educazione in tempo di pace): Useppe, infatti, è la nostra coscienza di fronte alla storia. Comencini semplifica volutamente i messaggi morantiani riducendoli a "quadri parlanti": uno sulla vitalità mal riposta, un altro sull'innocenza travolta e schiacciata, un altro ancora sull'ebraicità, sulla coerenza mancata ecc. Per mezzo dei filmati d'epoca in bianco e nero che s'alternano ai trascorsi dei personaggi fittizi, spiega ai semplici di spirito il rapporto tra la micro- e la macro-storia che non procedono parallele, bensì si incrociano: all'origine di ogni evento storico v'è un atto (o un atto mancato) umano.
\end{abstract}

Parole chiave: micro-storia, macro-storia, innocenza, bambini, cinema morale.

\section{The History of Morante on the Screen}

\begin{abstract}
This interpretation of the cinematographic version of the novel La Storia by Luigi Comencini is far from a re-signification of the literary text. We will prove that this director, who has a pedagogical intent but opposes the political use of the cinema (and pays attention to avoid the typical pitiability of the Neo Realism) faithfully respects the Morant's aims. He makes a film on the innocence threatened by death and violence during the war (at the same manner, innocence is threatened by education during the peace). Useppe is our own conscience in front of the history. Comencini deliberately simplifies the contents of Morante's novel, which are transformed in exemplary scenes: the first of them on the equivocations of vitality, the second on the destroyed innocence, the third on Jewishness, the fourth on incoherence, etc. Comencini explicates the relation between the micro-history and the macro-history, which intersect one to each other although they don't go ahead in parallel, using old documentary which alternate with the history of the main characters. The genesis of every historical event is an human act (or an human failed act).
\end{abstract}

${ }^{1}$ Dipartimento di Italianistica, Ulica Obozna 8, P-00 927 Varsava (Polonia). 
Key words: Micro-history, macro-history, innocence, children, moral cinema.

In questo articolo il testo del romanzo di Elsa Morante (1974) sarà posto sullo sfondo del discorso principale che verterà sull'adattamento filmico realizzato da Luigi Comencini (1986) per il piccolo schermo ${ }^{2}$. Concentrandoci sul film, vedremo come il regista, senza fare un torto al romanzo, rimane fedele essenzialmente a se stesso, alle proprie scelte estetiche e ideologiche, alla perenne preferenza per le sue tematiche, senza perciò venire meno ai messaggi che sottostanno al romanzo morantiano. Mentre la trasposizione cinematografica del precedente romanzo della Morante, realizzata da Damiano Damiani (Isola di Arturo, 1962) si rivela per molti versi un atto di fedeltà e un omaggio all'opera e alla figura della scrittrice (Serkowska, 2014), ad emergere dal film di Luigi Comencini, al pari dell'opera della scrittrice, è il regista - autore di altri film su bambini, un artista che ripete a più riprese lo stesso racconto con un'unica morale. Concentrato sull'infanzia, si sente chiamato a proteggerla raccontandola, e mentre ne esalta le doti - lavorando su La Storia - dice non solo che: «l'infanzia [ha] la caratteristica fondamentale di essere il "solo" momento di grande "libertà" per un individuo: il processo con cui l'educazione scolastica e familiare tende a soffocare questa libertà è drammatico» (Comencini 1974). Egli ci fa vedere come l'infanzia diventi la vittima predestinata della guerra che è l'immagine paradigmatica della violenza degli adulti in genere, e come ogni sforzo di sottrarre l'infanzia alla guerra e alla Storia sia destinato a fallire. Concorda in ciò con l'idea morantiana espressa nel romanzo: «Useppe è malato di epilessia, ma la sua morte è causata dall'impossibilità di vivere in questo mondo. Una morte emblematica» (Morante 1988 [1990]: 253); «Useppe è la nostra coscienza di fronte alla Storia» (Morante 1988 [1990]: 255).

Il regista lombardo, ricordiamo brevemente, esordito con il lungometraggio Bambini in città (1946), ha al proprio attivo numerosi film dedicati all'infanzia: L'incompreso (1967), Vita, infanzia e adolescenza di Giacomo Casanova veneziano del 1969, e gli adattamenti televisivi di Pinocchio (1971) e di Cuore (1983). La trasposizione de La Storia doveva coronare quel lungo percorso, durante il quale abbiamo visto Comencini mettere in scena sempre una coppia antitetica di ragazzi, spesso fratelli, di cui il minore appare un bambino angelico, che Paolo Mereghetti definisce un essere premorale, l'essenza dell'innocenza infantile, mentre quello maggiore rappresenta la coscienza socializzata e responsabilizzata, sebbene

\footnotetext{
${ }^{2}$ Premetto che in questo saggio non si parlerà dei rapporti di Elsa Morante con il mondo del cinema, rapporti che vanno dall'iniziale (o giovanile) piena fascinazione - Morante scrisse perfino dei soggetti e dei treatment - al rifiuto dell'establishment dei "padri farisei". L'argomento è stato esaurientemente trattato da Walter Siti, in occasione del suo lavoro su Pasolini (Siti 2006), e Marco Bardini ha da poco ultimato una monografia sui rapporti di Elsa Morante con il cinema. Io stessa ho dedicato un saggio all'adattamento cinematografico di un altro romanzo, Isola di Arturo (Serkowska 2014).
} 
entrambi, in definitiva, sono testimoni dell'insensibilità degli adulti (Mereghetti 2007: 172 ).

Occuparsi dei bambini e delle loro madri nel dopoguerra, dopo il 1946, come nota Tullio Masoni, era «come guardare al dramma delle famiglie mutilate in città semidistrutte e agli orfani», e ciò portava «in primo piano l'infanzia e, in generale, le vittime civili della guerra» (Masoni 2007: 33). Ma il fatto che il regista si cimentasse con il problema della distruzione causata dalla guerra, con un ritardo simile a quello con cui Morante si poneva l'argomento, permetteva innanzi tutto di presentare la guerra non come un evento contingente, di fresca memoria, causa di ferite ancora sanguinanti, bensì come una condizione umana universale: la barbarie ricorrente, insomma, d'accordo con l'ultima frase del romanzo morantiano: «.....e la Storia continua......» (Morante 1988 [1990]: 1029). Notò giustamente Cesare Garboli poco dopo che La Storia venne pubblicato: «il romanzo diventa terribile e tragico dopo la guerra, non durante» (Morante 2012: 505), e così anche a noi sembra che Comencini riesca a presentare la guerra come un dramma perenne a decenni di distanza dal conflitto mondiale.

In quali circostanze il regista si accingesse a girare il film omonimo del romanzo morantiano, ce lo ha spiegato Giorgio Gosetti, discutendo insieme di due lavori del regista: La Storia e Un ragazzo di Calabria, entrambi a metà strada tra committenza e libera ispirazione.

Comencini appare in tale prospettiva come un artista che ha cercato di sottrarsi all'obbligo di pronunciarsi sull'industria cinematografica, e di sottrarre il cinema al confronto con le problematiche e le limitazioni dell'industria filmica ${ }^{3}$. Gosetti interpreta inoltre quei due film come testimonianza di un regolamento dei conti con generi e tematiche, stereotipi e curiosità precedenti (Bildungsroman, infanzia, trasformazione sociale, contrapposizione città-campagna, la saga degli sconfitti, il sogno di un mondo migliore). Elsa Morante era poi in agonia durante le riprese, ma aveva accettato, dopo aver rifiutato numerose altre proposte, che Luigi Comenicini girasse il film. Lo studioso afferma che Morante lo fece per stima e simpatia, dopo aver visto le cassette con Le avventure di Pinocchio che Comencini le aveva portato in ospedale (Gosetti 1988).

L'ultima considerazione preliminare riguarda il fatto che la mia è una lettura della riduzione cinematografica per il piccolo schermo. La scelta stessa della televisione, così cara al regista, gli permette di raccontare a lungo, a diverse riprese, senza dover tagliare, elidere, cosa che, come ricorda Luciano De Giusti (Masoni 2007: 81-186), il regista detestava. Comencini odiava ridurre un testo lungo, mutilarlo per il cinema. Anche considerate le reazioni dei lettori comuni al lungo

${ }^{3}$ Secondo Mereghetti: «ancora negli anni Sessanta non si può parlare da noi di un cinema che si sottrae strategicamente ai condizionamenti dell'industria... Il cinema italiano ha saputo trovare degli accordi necessari con l'industria per non marginalizzarsi. Luigi Comencini, per esempio, ha alternato film "su commissione" (come L'incompreso), a film personali ma d'insuccesso, conquistandosi la vera libertà e autorialità», conclude lo studioso. («Premessa», Mereghetti 2007: 12). 
romanzo morantiano, contenute in lettere inviate all'autrice dopo la pubblicazione de La Storia, non si poteva che seguire il corso epico, lento, maestoso della storia raccontata dalla Morante in oltre seicento pagine, per non privare gli spettatori della compagnia di Useppe, Bella, Ida e di tutti gli altri ${ }^{4}$. Infine, fare un film per il piccolo schermo, metteva l'opera a portata di tutti, e anche in questo Luigi Comencini si rivela un artista attento e rispettoso di chi, al fine di rendere accessibile il libro al largo pubblico, insisteva su un prezzo di copertina bassissimo.

L'adattamento de La Storia (similmente a Le avventure di Pinocchio) riveste per Comencini un'importanza particolare. Il regista confessa che tra i suoi adattamenti, solo Le avventure di Pinocchio e La Storia erano libri che egli non detestasse, e quindi per questi due lavori non valse la regola secondo cui egli aborriva in genere i libri da cui traeva i suoi film - unico tratto comune dei suoi adattamenti, stando a quel che Comencini ammette (Comencini 2008: 251-268). Proprio trattandosi di due libri che egli amava molto, non aveva il coraggio di maltrattarli (Comencini 2008: 252). Nel caso de La Storia in specifico il regista era ben cosciente del pericolo di ricadere anacronisticamente nel neorealismo, facendone un torto al libro. Uno degli espedienti cui egli ricorre all'uopo, è il rifiuto di scegliere per il ruolo di Ida un'attrice sgraziata e brutta. Claudia Cardinale - spiega Comencini - permette che lo spettatore si identifichi con una protagonista graziosa, e che tale identificazione promuova l'empatia, dato che il romanzo di Elsa Morante richiedeva una partecipazione che non fosse solo pena e commiserazione (Comencini 2008: 253). Ida, nel romanzo morantiano, è una bella persona, seppur non nell'aspetto; ma «raccontati, anche i brutti possono sembrare bellissimi. Non così in un film, dove ciò che si vede viene prima di ogni altra cosa» (Comencini 2008: 255). Un altro baluardo eretto dal regista per premunirsi contro gli indesiderati caratteri neorealisti - i personaggi dei film neorealisti non sognano - è costituito dal ricorso regolare e sistematico alle scene dei sogni della protagonista. E la materia dei sogni "proiettati" sullo schermo è strumentale alla maggiore comprensibilità della storia che ci viene raccontata, sottolineando le motivazioni e le conseguenze dell'agire dei personaggi. I sogni della Ida morantiana costituiscono una parte importante della sua vita, spiegano le sue paure, i traumi, i desideri. E nel film di Comencini i sogni, anche se non moltiplicati, date le necessarie ellissi, paiono più numerosi nella vita

\footnotetext{
${ }^{4}$ Basti vedere la recente raccolta di lettere pubblicata a cura di Daniele Morante e con la collaborazione di Giuliana Zagra per accertarsi del desiderio espresso dai lettori: «Il suo stupendo libro ha un solo punto negativo: è troppo breve. Avrei voluto che durasse due o tre mila pagine o per sempre» (Morante 2012: 501). Altri giovani lettori informavano Elsa Morante di aver deciso all'unanimità di adottare La Storia come ufficiale libro sussidiario di lettura che sarebbe pertanto rientrato nel loro programma di esame (Morante 2012: 503 e 504). Il senso di gratitudine e gioia che il libro suscita, rendendo difficile ogni discorso critico, era del resto provato da lettori per niente comuni, come Anna Maria Ortese («quando il libro è finito $[\ldots]$ della letteratura non ci ricordiamo [...], ma sì del dolore umano» (Morante 2012: 502) e Giorgio Agamben «solo gratitudine e gioia sono una risposta adeguata» (Morante 2012: 515).
} 
di veglia. Quasi come i documentari in bianco e nero, si presentano a scadenze regolari. Diversamente dal romanzo è poi quasi assente, visto che al regista questi contenuti non interessano, l'impianto psicanalitico palese nel romanzo, con ovvi riferimenti al Familienroman su cui è improntata l'intera opera morantiana. Sullo schermo i sogni sono legati agli eventi vissuti da Ida in veglia. "Scattano" in prossimità dei momenti più tesi della vita di Ida con uno scopo preciso, quello di spiegare le ragioni dei dubbi, chiarire le decisioni da prendere, illuminare il percorso da seguire. Lo stupro perpetrato dal soldato tedesco, che Ida vive come un atto di ricatto volto contro la sua ebraicità, provoca un sogno in cui compare la sua madre ebrea che spiega alla piccola Ida che il suo cognome ebraico non è Almagìa, bensì Almagià, e di aver fatto battezzare tutti i figli, visto che gli ebrei sono un popolo predestinato all'odio perenne. Invece nel momento in cui la protagonista scopre di essere rimasta incinta dallo stupro, si vede passeggiare con suo padre che canta un'aria dell' $A i d a^{5}$, coprendola con un lembo del mantello, facendola quasi scomparire là dentro come fanno i maghi o i prestigiatori, per proteggerla dalle poche gocce di pioggia estiva. Quando inizia a partorire, e le si rompono le acque sul finir della notte, le compaiono in sogno dei cadaveri trainati su carrette, e dall'ultima sporge una manina infantile: spia del desiderio represso di sbarazzarsi della gravidanza - frutto di violenza, o forse prodotto della paura di morire nel parto o di partorire un feto morto. Si tratta di un'immagine di morte, la cui ombra incombe già sull'isola di Arturo (la cosa che Arturo teme di più al mondo è la morte), che qui coincide con Useppe, il personaggio che ha più disperatamente bisogno di amore (eloquente il gesto con cui egli accarezza la mano di Davide, e si stringe al petto di Nino), che è capace di amare il mondo, gli animali, gli umani, e di attaccarvisi in modo ossessivamente assoluto. Allo stesso tempo Useppe non riesce a sottrarsi alla triade di guerra-storia-morte. Nato come frutto della violenza, è presto costretto a vivere una serie di morti ("volano via" uno dopo l'altro Blitz, Giuseppe, Nino, Davide, Scimò), compresa la propria, causata dal "grande male". Prima della sua morte, tuttavia, abbiamo ancora un altro sogno che la preannuncia. Dopo che Useppe ha visto su un giornale le foto che accompagnano il servizio chiamato «La verità sulla barbarie nazista», Ida dice al figlio: «perché guardi questa roba? È brutta, strappiamola», e di notte fa un sogno in cui vede una montagna di scarpe (in luogo del mucchio di ossa e teschi umani, appena visti sulla foto del giornale) e cerca, senza trovarla, l'altra scarpetta di Useppe. Poco dopo assistiamo al risveglio notturno della madre che non trova il suo piccolo, colto da un ultimo e fatale accesso. La madre con una scarpa in mano, trova il figlio seduto su una sedia, lo abbraccia, cerca di calmarlo. Gli altri due sogni di Ida sono legati a Nino, Davide

${ }^{5}$ L'Aida verdiana, ricordiamo, vive come schiava e la sua vera identità rimane nascosta. Liberata dalla prigionia da suo padre, muore insieme all'amato di una morte atroce. Il motivo dell'opera verdiana, presente nel romanzo morantiano, persegue lo stesso scopo nel film: rievoca il padre amato ma scomparso e allude al destino glorioso sospirato, ma mancato in vita dalla protagonista, ostaggio della storia, costretta comunque a nascondere la sua vera identità. 
e Günther. Alla dichiarazione gagliarda di Nino diventato partigiano «se ne vedo uno in piedi, di tedeschi, lo stendo», segue il sogno di Ida che raffigura Günther morente e prefigura un altro corpo in agonia, quello del soldato tedesco che Davide avrebbe ucciso a calci. E dopo la morte di Nino la madre sogna la propria morte: indossa gli occhiali da sole del figlio (rotti) e lo vede su una barca a remi impassibile alle sue grida. Ida, che si sente pertanto afona, impotente, con le braccia legate con una corda che le deve impedire di nuotare, entra nell'acqua per annegare. Chiama ancora il figlio senza emettere nessun suono, come succede nel sogno. Nino non la sente, Ida scompare sott'acqua.

L'idea che nel film di Comencini vi sia un'operazione di ri-semantizzazione vera e propria guida, al contrario, la studiosa Tiziana Jacoponi, convinta che vi fosse di mezzo un tradimento metodico e costante da parte del regista nei confronti dello spirito del romanzo, e che il tradimento fosse legato al fatto che Comencini era «lo specialista dei bambini» (Jacoponi 2000). La studiosa muove dal paragone di questo con un altro celebre adattamento (Il Gattopardo), e constata che esso, diversamente da La Storia di Comencini, ha riportato un clamoroso successo. E, come per spiegare il fallimento de La Storia, Jacoponi elenca una serie di possibili infedeltà, tra cui ne spiccano tre che brevemente illustro. Il film mancherebbe del "miserabilismo", dello squallore e della desolazione, viceversa presenti in maniera ossessiva nel romanzo, dove al degrado esterno si affianca un degrado interno dei personaggi («Useppe è sempre mal vestito o vestito di stracci, è sempre sporco e trasandato», Jacoponi 2000: 119). In secondo luogo, il personaggio di Ida, che nel romanzo appare sin dall'inizio invecchiata precocemente, imbruttita, ridotta a una femmina di un animale che perlustra le strade di Roma in cerca di viveri per i propri piccoli, è interpretato da Claudia Cardinale, attraente e ben curata, e che, nella scena dello stupro, mostra le cosce tonde e ben tornite. Bella, serena, materna, sorridente, tutt'altro che tesa, impaurita e mortificata, la diva non renderebbe giustizia al personaggio dell'Ida morantiana, dal corpo denutrito, informe, dal petto sfiorito, coperto di un cappottino da vecchia, consunto e stracciato. Infine, il colore degli occhi di Useppe, che nel romanzo è turchino («colore turchino del suo scandalo», cioè dello stupro perpetrato dal soldato tedesco, ma anche di un colore che riproduce «quell'altro turchino che non pareva nato dalla terra ma dal mare»), nel film diventa marrone.

Fatta eccezione per il colore degli occhi di Useppe - un dettaglio importante, sì, ma solo per i morantisti più oltranzisti, le colpe di Comencini sembrano piuttosto veniali. La scelta di un'attrice come Claudia Cardinale è stata giustificata dal regista in maniera più che convincente. Quanto all'insufficiente "miserabilismo", il terrore di cadere nella vecchia maniera neorealista, esecrata dalla scrittrice stessa, ha sicuramente influito sulla scelta di abiti comunque non ridotti a stracci, e a personaggi non propriamente cenciosi.

Notevole invece la trovata del regista che riproduce perfettamente l'ambiguo rapporto tra la micro- e la macro-storia del romanzo. Comencini ricorre ai filmati 
d'epoca in bianco e nero su cui conviene brevemente soffermarsi. Ricorrendo ai documentari d'epoca ${ }^{6}$, il regista riesce perfettamente a tradurre coi mezzi filmici l'operazione morantiana che vede alternati alla narrazione delle vicende di personaggi romanzeschi, brevi sintesi della Storia in fieri, scritti in corpo minuscolo e posti tra i capitoli. Gli spezzoni in bianco e nero rappresentano tre tipi di eventi. Il primo - la cui funzione è quella di incardinare la vicenda, incardinarla nel tempo e nello spazio - sono i discorsi del Duce in piazza. Nel primo discorso Mussolini esalta il destino degli italiani «scritto nei cieli» e le «decisioni irrevocabili»; nell'altro - parla dell'Italia e della Germania sempre più strettamente alleate, ora affiancate anche dall'eroico Giappone. Ai discorsi seguono le grida estasiate delle masse accorse ad ascoltarli ${ }^{7}$ e il commento di una voce extradiegetica, salmodiante. La voce elenca come litanie i dati storici che s'accompagnano all'evento appena ritratto nelle immagini (l'Italia dichiara la guerra alla Francia e alla Gran Bretagna, ecc.). Un altro tipo di fotogrammi, che raffigura la marcia dei giovani fascisti, si ricollega con la decisione del personaggio Nino, impaziente di partire per il fronte e di fare la guerra. Le scene della sconfitta dell'offensiva delle truppe italiane in Russia, sprovviste di mezzi elementari per la sopravvivenza, di molti soldati dispersi e sepolti nella steppa gelata, anticipano la morte vicina di questo personaggio, in tutto fedele al Nino morantiano, un giovane che brucia l'adolescenziale ansia di vivere, buttandosi nella lotta e che cambia bandiera passando da fascista a partigiano a contrabbandiere ${ }^{8}$. Infine, nell'ultimo gruppo di filmati è la voce fuori campo a guidarci, e ad annunciare la fine della guerra, riportando cifre, date, di nuovo in guisa di una litania spassionata: «con la morte violenta di Hitler e di Mussolini, e con le bombe di Hiroshima e Nagasaki [...] morti, feriti, dispersi...». La Storia continua, insomma, esattamente come nel finale del romanzo morantiano. Comencini sembra aver reso lo stesso complesso rapporto

${ }^{6}$ Quei filmati originali includono anche i proclami e i manifesti su cui si annunciano i provvedimenti delle Leggi Razziali e le disposizioni del Governatorato di Roma sui cittadini appartenenti alla razza ebraica, documenti a cui la Morante dà un notevole rilievo nel romanzo, e che trovano nel film un loro corrispettivo. Ida, impaurita, si ferma a leggere e fugge via mormorando tra sé: «sempre lo stesso».

${ }^{7}$ In contrasto con la reazione entusiasta delle masse del documentario, e a suggerire forse che anche i documenti originali possono non dire tutta la verità (l'idea della stessa Morante (cfr. Serkowska 2003: 185-191), vediamo il comportamento di Giuseppe, il suonatore di organetto che disdegna la moneta regalata dal soldato tedesco, e del ragazzo dell'oste che non serve il tedesco al tavolo, ma appoggia la bottiglia sul banco, incrocia le braccia e con aria di sfida fa un passo indietro, costringendo il tedesco ad alzarsi e a servirsi da solo. Infine Ida e Useppe e molti altri, impauriti dalla guerra, sono costretti a soffrirne le conseguenze, o a mostrare la resistenza, come gli sfollati fatti alloggiare nello stanzone di Pietralata che buttano giù il busto del Duce, vi sputano e orinano sopra.

${ }^{8} \mathrm{Nel}$ film Nino muore ma non "del tutto". Al mondo lascerà una creatura, un altro figlio di guerra come Useppe. Questa invenzione di Comencini è con ogni probabilità ispirata a L'Isola di Arturo in cui all'Arturo che abbandona l'isola si sostituisce il suo piccolo fratellastro, Carmine-Arturo. 
che esiste tra la storia in grande, o quella scritta sui libri, e la storia del singolo. All'apparenza le due procedono ciascuna per conto proprio, e la storiografia non riflette le vite singole, ma il romanzo e il film ci fanno vedere che separarli a nulla serve, che all'origine di ogni atto storico v'è un atto umano. L'essere umano è vittima e carnefice a turni alternati. Come Günther, violentatore ma a sua volta travolto dalla guerra e vittima, o come Davide, giovane idealista ribelle, ebreo anarchico non violento, ma al contempo un borghese che vive di rendita, un drogato, infine un omicida. I filmati si rivelano pertanto una specie di corrispettivo diurno dei sogni di Ida: palesemente intrecciati con gli eventi della diegesi, non evidenziano la diversità tra la storia privata e quella ufficiale, ma piuttosto un loro intrinseco legame.

La guerra, il prodotto simbolico del nostro operare nella storia, sintesi mostruosa dell'agire umano, si ripercuote fortemente sulla vita dei singoli, nel romanzo come nel film, e La Storia si rivela una toccante - e paradigmatica - odissea di una madre dotata di pochi mezzi, ma armata di un istinto di tigre verso il proprio cucciolo ${ }^{9}$, una madre che affronta in modo autolesionista la carestia, il terrore e la morte ai tempi della guerra. Eloquente pertanto e brillante mi pare - per la sua aderenza e fedeltà allo spirito del romanzo - la scena finale del film in cui, senza colonna sonora, gli spettatori, insieme ai vicini e ai carabinieri, rimangono sulle spine, col fiato sospeso, allibiti di fronte alla porta chiusa, sconvolti da cotanta, afona, atrocità. Comencini trasferisce sul cane Bella le doti della morantiana madre-tigre che minacciava chiunque tentasse di privarla del corpicino, ormai esangue, del piccolo Useppe, al punto che «si rese necessario abbattere la bestia» (Morante 1988 [1990]: 1019).

Il messaggio trasmesso da Morante e da Comencini è unanime anche perché entrambi sono convinti che l'arte non deve prestarsi a fini politici. Era noto il silenzio morantiano anche durante gli anni di piombo (la lettera alle BR rimasta inedita) e il rifiuto di pronunciarsi sull'attualità in genere, di lasciarsi coinvolgere e usare a servizio di qualche idea politica. Comencini, mosso forse da altre motivazioni, già confrontandosi con un'opera politica quale a suo tempo era $L a$ ragazza di Bube, un'opera di critica della recente storia italiana, evitò di "scrivere" una lettera politica. Similmente accade nel film qui in disamina. In reazione ai discorsi ingarbugliati di Davide (che non si rassegna, e a guerra finita ripete come un mantra: «la storia è tutta un'oscenità», «l'ultima guerra è stata la peggiore di tutte»), gli altri avventori dell'osteria si rivelano desiderosi di dimenticare la guerra e andare avanti, e ribattono: «il morto giace, il vivo si dà pace». Seppur convinto che, se il cinema fosse una trascrizione di un'opera politica, sarebbe un incontro

${ }^{9}$ La parabola delle tigre, tra le tante raccontate ne La Storia, la più atroce, rende la condizione in cui Ida è ridotta, avendo trasferito tutti i suoi istinti vitali su Useppe, a cui non può dar più nulla da mangiare: «Si racconta di una tigre che, in una solitudine gelata, si sostenne assieme ai propri nati leccando, per parte sua, la neve; e distribuendo ai piccoli dei brandelli di carne che lei stessa si strappava dal proprio corpo coi denti» (Morante 1988 [1990]: 643). 
futile, «sarebbe come illustrare un romanzo» (Pasolini 1978: 268-271); che il cinema abbia una sua poetica e produca opere di narrazione autonome («...secondo me un film è sempre un'altra cosa rispetto al libro dal quale è tratto», Comencini 2008: 74-92), il regista, nel caso de La Storia, ci consegna un film che presta mirabilmente fede al romanzo da cui è tratto. L'unico procedimento o trattamento cui il testo letterario viene sottoposto, è quello di esplicitazione o chiarificazione per semplificare al massimo la diegesi. Il regista dipana il racconto secondo le regole della linearità, essendogli cara in genere l'unità d'azione che egli ritiene essenziale in un'opera che si vede (Gosetti 1988: 94-100). Di conseguenza il film assume un carattere quasi pedagogico, è galleria di "quadri parlanti", esattamente come Elsa Morante desiderava che l'arte potesse comunicare ai semplici di spirito. Vediamo ora qualche esempio di tale propedeutica semplificazione che va di pari passo con l'enfasi sugli elementi-chiave del racconto: l'ebraicità di Ida, la vitalità di Nino, la coerenza di Davide e l'innocenza di Useppe.

Il tema dell'ebraicità. Quando il soldato-bambino Günhter, violentatore ma anche disperatamente alla ricerca di un calore materno, segue Ida e si introduce in casa sua, lei ripete: «Ho fatto denuncia al comune. Sono un'insegnante di ruolo» e tre volte: «Io non ebrea». Si va dal terrore di essere scoperta, alle scene delle deportazioni cui Ida assiste, scene atroci alla stazione Tiburtina e vagoni bestiame con dentro stipati gli ebrei, alle voci dell'eccidio lontano degli ebrei che risuonano nei discorsi allucinati della gattara del ghetto. Faccia truccata come un clown o personaggio di Fellini: giustamente, perché la gattara è un personaggio surreale con le sue profezie da indovina, che suonano come eresia o follia pura. Predice gli eventi ancora da consumarsi: («una strage peggio di quella di Erode»), supplica le donne ebree, e ora anche Ida, già incinta: «non li fate nascere», infine confonde $\mathrm{i}$ gatti con gli ebrei. Mentre li conta e nota che essi scompaiono poco per volta, bofonchia: «li prendono, li portano via...». Eppure, per partorire Ida sceglie di recarsi nel ghetto, nella casa della levatrice con vista sulla Sinagoga. E dopo le deportazioni andrà a percorrere le strade del ghetto già vuote - le finestre spettrali, $\mathrm{i}$ cortili abbandonati.

La vita di Nino è presentata in modo esemplare: quel che succede al giovane personaggio, dimostra che cosa succede ai ragazzini che si mettono dalla parte della guerra-storia. La vitalità, o il vitalismo del quindicenne primogenito di Ida viene smorzata, stroncata dall'impatto con la storia nella quale egli si precipita con tanta irrequietezza. Nino si specchia tendendo i muscoli e urlando «vita vita vita», e lo spettatore intende: «guerra, guerra, guerra», o «morte, morte, morte». E sono sue, di Nino, le svolte apparenti, che non sono delle vere svolte, esattamente come nella storia che è e rimane sempre uguale. Il cambiar bandiera di Nino viene perciò reso in modo semplificato, pur con citazioni più dirette dal romanzo: da giovane fascista, impaziente di compiere i tre mesi di preparativi al campo Dux e partire in guerra (compare vestito da squadrista, fa un saluto romano con un altro "camerata" che passa a prenderlo; esalta la "presa di Parigi": «Parigi è nostra voglio anna' a vede' com'è fatta»; poi lo vediamo partire al fronte russo, salutare la madre con un entusiastico «pum pum pum»); diventa di colpo partigiano e mentre ricompare a 
Pietralata saluta gli sfollati con un «buonasera compagne e compagni», si presenta come Asso di Cuori a fianco di un altro compagno, Quattro Punti. Infine si dà al contrabbando, e arriva perciò prima in motocicletta portando cioccolata, sigarette $\mathrm{e}$ denaro, poi in una Jeep con un cane. Le sue "conversioni" scandiscono i tre atti della commedia della guerra: «la guerra era tutta una commedia», lo sentiamo dire prima del finale tragico.

Che ogni tentativo di rimanere dalla parte della non violenza, non azione, pacifismo, come ogni tentata purezza, siano presto destinati ad arrendersi di fronte all'evidenza della storia, lo dimostra la vicenda di Davide, sedicente anarchico. In un momento lo sentiamo dire: «la vera anarchia non ammette violenza; tutto il male sta nella violenza»; «il potere è la lebbra del mondo...»; «il guadagno, la proprietà, la gerarchia sono depravazioni...». Nella sequenza immediatamente successiva egli è venuto a sapere della deportazione e morte dei suoi familiari, butta alle ortiche le idee di prima, convinto che «la non violenza non serve a nulla». Pecca della stessa mancanza di coerenza anche Ida che prima redarguisce il figlio Nino per il furto di viveri e in seguito cede alla fame e mangia insieme a Nino delle salsicce rubate. Più tardi raccoglie la farina rubata da un camion e ruba un pezzo di carne... La coscienza, quella che a dir di Davide, è la cosa di cui è dotata solamente «la creatura umana» («si nasce bianchi, ebrei o neri per puro caso, ma non si nasce creature umane per caso»), la nostra capacità di pensare, di ragionare, di sentire, di agire ragionevolmente, diventa un peso inutile di cui Davide, invano, cercherà di liberarsi facendo un faticoso lavoro manuale.

Infine abbiamo Useppe, "tradotto" ad uso dello spettatore di massa, definito dalla madre all'inizio «troppo buono», da Davide chiamato «idiota» e che non capisce la malvagità di questo mondo, che muore ucciso dal male e dalla violenza presenti nella vita e storia umane.

Sul set de La Storia, che non ri-semantizza ma piuttosto appiana e chiarisce il messaggio morantiano, si incontrano due elementi dei film di Comencini. Il suo è un cinema morale, etico (Pistagnesi 2007), pedagogico, di chi era comunque determinato a combattere una sua battaglia civile anche quando girava una cronaca dei suoi tempi, un cinema caratterizzato dall'amore per l'infanzia. Il regista riesce a evitare di «scrivere una lettera politica» seguendo Elsa Morante, limitandosi ai mezzi che la scrittrice gli ha suggerito nel suo romanzo ${ }^{10}$. Il film ritrae una società di famiglie mutilate, portatrici dei lutti della guerra, di mariti e padri assenti o lasciati ai margini, di orfani, di madri singole, di fidanzate incinte e vedove, di figli nati da stupri, di altri personaggi travolti dalla guerra. Sopra tutti trionfa la morte, la prima protagonista che vanifica ogni atto umano nella storia, ogni sforzo di bontà, di purezza, di amore e di non violenza. Un'idea peregrina nella produzione di un artista il cui cuore è tenero e che ha sempre preferito i buoni sentimenti? Sì se si pensa che il mondo adulto è sempre e comunque ostile, che da esso sia impossibile

${ }^{10}$ Secondo Tullio Masoni, i film di Comencini (Masoni si riferisce a Le avventure di Pinocchio) sono «delle favole neorealiste senza neorealismo» (Masoni 2007: 39). 
cacciare la violenza e la morte, perché esse ritornano sotto mentite spoglie e travolgono l'innocenza angelica degli Useppe di questo mondo... Se tuttavia si ricorda l'epigrafe del romanzo morantiano, «Tutti i semi sono falliti, eccettuato uno, che non so cosa sia, ma che probabilmente è un fiore e non un'erbaccia» (Morante 1988 [1990]: 1031), si è rinfrancati e la speranza non muore ancora.

\section{RIFERIMENTI BIBLIOGRAFICI}

BARDINI, Marco (2014): Elsa Morante e il cinema, Pisa, ETS:

COMENCINI, Luigi (1974): «Entretien avec Luigi Comencini», in Lorenzo Codelli, (ed.), Positif, 156, p. 16.

COMENCINI, Luigi (2008): La Storia. Dal romanzo al film, in Arpà, Adriano (ed.): Il cinema secondo me. Scritti e interviste (1974-1992), Milano, Il Castoro cinema, pp. 251-268.

GoSETTI, Giorgio (1988): La storia di Ida e di Mimì, in Luigi Comencini, Milano, Il Castoro Cinema, 13/5, pp. 94-100.

JACOPONI, Tiziana (2000): «La Storia: un libro, un film», in Narrativa, 17, pp. 117122.

LuCAMAnTe, Stefania (ed.) (2014): Rethinking Subjectivity, History and the Power of Art: Elsa Morante's Politics of Writing: Madison, Fairleigh Dickinson University Press.

MASONI, Tullio (2007): Stato di famiglia. La battaglia civile (e amorosa) di Luigi Comencini, in Arpà, Adriano (ed.): Luigi Comencini. Il cinema e il film, Venezia, Edizione Marsilio, pp. 31-34.

MereghetTi, Paolo (2007): Incompreso, in Arpà, Adriano (ed.): Luigi Comencini. Il cinema e il film, Venezia, Edizione Marsilio.

MoRANTE, Elsa (1988 [1990]): Opere, a cura di C. Cecchi e C. Garboli, Milano, Mondadori, 2 voll.

MORANTE, Elsa (2012): L'amata. Lettere di e a Elsa Morante, in Morante, Daniele (ed.) Milano, Einaudi.

PASOLINI, Pier Paolo (1978): L'intellettuale impegnato (1964), in Ferretti, Gian Carlo (ed.): Le belle bandiere, Roma, Editori Riuniti.

Pistagnesi, Patrizia (2007): Il cinema etico di Luigi Comencini, in Arpà, Adriano (ed.): Luigi Comencini. Il cinema e il film, Venezia, Edizione Marsilio, pp. 17-30.

SERKOWSKA, Hanna (2003): Uscire da una camera delle favole, Rabid, Kraków.

SERKOWSKA, Hanna (2014): "Arturo in the World of the 'Pharisee Fathers': Considerations on the Cinematic Adaptation of Arturo's Island ", In Lucamante, Stefania (a cura di): Rethinking Subjectivity, History and the Power of Art: Elsa Morante's Politics of Writing: Madison, Fairleigh Dickinson University Press.

SITI, Walter (2006): Elsa Morante and Pier Paolo Pasolini, in Lucamante, Stefania / Wood, Sharon (ed.): Under Arturo's Star: The Cultural Legacies of Elsa Morante, West Lafayette, Purdue UP, pp. 268-88. 